\title{
A Note on the Generalised Measures of Risk Aversion*
}

\author{
TAPAN BISWAS
}

\author{
Department of Economics, University of Hull, Hull, United Kingdom \\ Received March 10, 1981; revised November 18, 1981
}

\begin{abstract}
This paper is concerned with generalised scalar measures of risk aversion. A measure $R$ which may meaningfully be applied to both unidimensional risks (risk in income or wealth) and multidimensional risks has been constructed. In case of identical preferences, we have also constructed an alternative measure of risk aversion $R^{*}$ which is shown to be related to the Khilstrom-Mirman measure. This relationship explains the nature of the Khilstrom-Mirman measure. Journal of Economic Literature Classification Numbers: 021, 022, 026.
\end{abstract}

\section{INTRODUCTION}

There have been quite a few generalisations of the Arrow-Pratt $[1,7]$ measures of risk aversion in the context of decisions involving $n$ commodities. Some of these measures deal with unidimensional risks alone (e.g., risk in income or wealth) while others deal exclusively with multidimensional risks. The natures of the unidimensional and the multidimansional risk problems are not similar. In this note, we shall construct a measure $R$ which may be meaningfully used in both cases. For multidimensional risks, any comparison of risk averseness requires the underlying preference orderings to be identical. In this case, we shall also develop an alternative measure of risk aversion $R^{*}$ which shall be shown as related to the Khilstrom-Mirman [6] measure $R_{\mathrm{KM}}$. The relationship between $R^{*}$ and $R_{\mathrm{KM}}$ explains the nature of the Khilstrom-Mirman measure in the multidimensional case.

\section{A Measure of Risk Aversion}

Consider an individual maximising his utility subject to a budget constraint. The utility function $u(x), x \in \operatorname{Int}\left(\Omega^{n}\right)$, is twice differentiable and

\footnotetext{
* I would like to thank the referee of this journal for helpful comments.
} 
strictly concave, and the associated Hessian matrix $U$ is negative definite. Let $p$ and $x$ denote the price and the commodity vectors, respectively. The sign " "denotes the transpose of a vector or a matrix as the case may be. The consumer is maximising utility $u(x)$ subject to the budget constraint $p^{\prime} x=z$. The well-known first-order conditions are

$$
u_{i}(x)=\lambda p_{i} ; \quad p^{\prime} \cdot x=z,
$$

where $\lambda$ stands for the marginal utility of income. Let us write the associated "indirect utility function" as $v=v(p, z)$. Since $U$ is negative definite, it is easy to show that $v$ is concave in $z$, i.e., the consumer is risk averse with respect to risks in income,

$$
\begin{gathered}
v_{z}=\lambda>0, \quad v_{2 z}=-|U| /|S|<0 ; \\
|S|=\left|\begin{array}{cc}
0 & p^{\prime} \\
p & U
\end{array}\right|=\frac{1}{\lambda^{2}}\left|\begin{array}{cc}
0 & \tilde{u}^{\prime} \\
\tilde{u} & U
\end{array}\right| \quad \text { and } \quad \tilde{u}=\left[\begin{array}{c}
u_{1} \\
u_{2} \\
\vdots \\
u_{n}
\end{array}\right] .
\end{gathered}
$$

Lemma 1. We have $v_{z z}=\lambda^{2}\left(\tilde{u}^{\prime} U^{-1} \tilde{u}\right)^{-1}$.

Proof. We have $v_{z z}=-|U| /|S|$. Both $U$ and $S$ are symmetric matrices;

$$
|S|=-\lambda^{-2} \cdot \sum_{i} \sum_{j} u_{i} u_{j} U_{i j}
$$

Here $U_{i j}$ is the co-factor associated with $u_{i j}$. Remembering $U_{i j}=U_{j i}$, we get $-|S| /|U|=\lambda^{-2} \cdot \tilde{u}^{\prime} U^{-1} \tilde{u}$. Our proposition follows immediately from Eq. (2).

Q.E.D.

In the one-commodity case, $x$ and $z$ are expressed in the same units $(p=1)$ and $v=v(z)$. The well-known Arrow-Pratt measures of absolute and relative risk aversion are

$$
R_{\mathrm{A}}=-v_{z z} / v_{z} ; \quad R_{\mathrm{R}}=-z \cdot v_{z z} / v_{z} .
$$

In the $n$-commodity case, it is clearly desirable that a change in the monetary or utility units should not affect the measures of risk aversion, which should satisfy the following conditions:

(C1) The measures of risk aversion should be invariant to any proportionate change in $p$ and $z$.

(C2) The measures of risk aversion should remain unaffected by any linear transformation of the utility function (Arrow-Pratt condition). 
Using Lemma 1 and Eq. (3) we obtain the generalised measure of relative risk aversion due to Hanoch [4]

$$
R_{\mathrm{R}}=-\tilde{u}^{\prime} x / \tilde{u}^{\prime} U^{-1} \tilde{u}
$$

From Lemma 1 and Eq. (3), we also obtain

$$
R_{\mathrm{A}}=-v_{z z} / v_{z}=-\lambda / \tilde{u}^{\prime} U^{-1} \tilde{u},
$$

which, unfortunately, does not satisfy $(\mathrm{C} 1)$ and can not be used as a measure of absolute risk aversion in the $n$-commodity case. However, from Eq. (1) we get

$$
\lambda=\tilde{u}^{\prime} \beta / p^{\prime} \beta \quad \text { and } \quad P=p^{\prime} \cdot \beta
$$

where $\beta^{\prime}=\left(\beta_{1}, \beta_{2}, \ldots, \beta_{n}\right), \sum \beta_{l}=1$, is an arbitrary row vector. With a proper choice of weights $\beta_{i}, P$ may be called a price index. Using Eqs. (5) and (6), we define

$$
R=-\tilde{u}^{\prime} \beta / \tilde{u}^{\prime} U^{-1} \tilde{u}=-P \cdot v_{z z} / v_{z},
$$

where $R$ may be treated as a measure of absolute risk aversion. Since $\sum \beta_{i}=1, R$ reduces to $R_{\mathrm{A}}$ in one commodity case. The measure $R$ satisfies both $(\mathrm{C} 1)$ and $(\mathrm{C} 2)$ and may also be shown as positively related to risk premium in real terms, using $P$ as a price deflator.

Consider individuals 1 and 2 whose preference orderings are identical (same indifference maps) but their (cardinal) utility functions, denoted by $u_{1}(x)$ and $u_{2}(x)$, are different. Using $R$ as a measure of risk aversion, if individual 1 is relatively more risk averse for all $x$, then we can show that $u_{1}(x)$ is more concave than $u_{2}(x)$. Since the preferences are identical, we may write $u_{1}=g\left(u_{2}\right)$. The function $u_{1}(x)$ is said to be more concave than $u_{2}(x)$ iff $g^{\prime \prime}<0$. Since both $u_{1}$ and $u_{2}$ are utility functions, $g^{\prime}>0$. Let $R_{1}$ and $R_{2}$ denote the values of $R$ with respect to the individuals 1 and 2. The following theorem is stated in terms of $R$; it could have been stated also in terms of $R_{\mathrm{R}}$ since $R_{1}>R_{2}$ iff $R_{\mathrm{R}}^{1}>R_{\mathrm{R}}^{2}$ for all $x, x \neq 0$.

THEOREM 1. If $u_{1}(x)$ and $u_{2}(x)$ are defined over the same preference ordering, $u_{1}(x)$ is more concave then $u_{2}(x)$ iff $R_{1}>R_{2}, \forall x$.

Proof. Since the preferences are identical, $u_{1}=g\left(u_{2}\right)$. Therefore,

$$
\begin{aligned}
\tilde{u}_{1} & =g^{\prime} \tilde{u}_{2}, \\
U_{1} & =g^{\prime \prime} \tilde{u}_{2} \tilde{u}_{2}^{\prime}+g^{\prime} U_{2} .
\end{aligned}
$$


From Eqs. (8) ad (9), by simple manipulations we get,

$$
\begin{gathered}
\tilde{u}_{2}^{\prime} U_{2}^{-1} \tilde{u}_{2}=\frac{g^{\prime \prime}}{\left(g^{\prime 2}\right)}\left(\tilde{u}_{1}^{\prime} U_{1}^{-1} \tilde{u}_{1}\right)\left(\tilde{u}_{2}^{\prime} U_{2}^{-1} \tilde{u}_{2}\right)+\frac{1}{g^{\prime}} \tilde{u}_{1}^{\prime} U_{1}^{-1} \tilde{u}_{1} . \\
(-)
\end{gathered}
$$

Note, $g^{\prime}>0$ and $U^{-1}$ is negative definite. Therefore, both $\tilde{u}_{1}^{\prime} U_{1}^{-1} \tilde{u}_{1}$ and $\tilde{u}_{2}^{\prime} U_{2}^{-1} \tilde{u}_{2}$ are negative. Consequently,

$$
g^{\prime \prime}<0 \quad \text { iff } \quad \tilde{u}_{2}^{\prime} U_{2}^{-1} \tilde{u}_{2}<\frac{1}{g^{\prime}} \tilde{u}_{1}^{\prime} U_{1}^{-1} \tilde{u}_{1}, \quad \forall x .
$$

From Eq. (8) we obtain $g^{\prime}=\tilde{u}_{1}^{\prime} \beta / \tilde{u}_{2}^{\prime} \beta$ which together with Eq. (11) proves that $g^{\prime \prime}<0$ iff $R_{1}>R_{2}, \forall x$.

Q.E.D.

Theorem 1 is significant. It implies that $R$ may be treated as a measure of risk aversion in the case of multidimensional risk, i.e., a risk in $x^{\prime}=$ $\left(x_{1}, x_{2}, \ldots, x_{n}\right)$. If $R_{1}>R_{2}$ for all $x$, it follows that $u_{1}(x)$ is more concave than $u_{2}(x)$ and individual 1 may be said to be more risk averse than individual 2 (See Khilstrom and Mirman [6]).

\section{An Alternative Measure of Risk Aversion}

Let us have a closer look at the case of identical preferences. We shall show that in case of identical preferences, it is possible to construct an alternative measure of risk aversion (multidimensional risk) defined as

$$
R^{*}=-\left[(-1)^{n}|U|\right]^{1 / n} / \tilde{u}^{\prime} U^{-1} \tilde{u} .
$$

THEOREM 2. If $u_{1}(x)$ and $u_{2}(x)$ are defined over the same preference ordering, $u_{1}(x)$ is more concave than $u_{2}(x)$ iff $R_{1}^{*}>R_{2}^{*}, \forall x$.

Proof. Consider Eq. (9). Since $\tilde{u}_{2} \tilde{u}_{2}^{\prime}$ is a positive matrix and $g^{\prime}>0$, $g^{\prime \prime}<0$ iff $U_{1}<g^{\prime} U_{2}$. Therefore,

$$
g^{\prime \prime}<0 \quad \text { iff } \quad\left[(-1)^{n}\left|U_{1}\right|\right]^{1 / n}>g^{\prime}\left[(-1)^{n}\left|U_{2}\right|\right]^{1 / n} .
$$

From Eqs. (11)-(13) we get

$$
g^{\prime \prime}<0 \quad \text { iff } \quad R_{1}^{*}>R_{2}^{*}, \quad \forall x . \quad \text { Q.E.D. }
$$

Consider the measure of risk aversion due to Khilstrom and Mirman [6]. 
In case of multidimensional risk, we can translate their measure in terms of our notation as

$$
R_{\mathrm{KM}}=\frac{(-1)^{n}|U|}{\left\{(-1)^{n} \lambda^{2}|S|\right\}^{n / n+1}}
$$

The following theorem proves that the Khistrom-Mirman measure, in the $n$ commodity case, is related to $R^{*}$.

THEOREM 3. We have $R_{\mathrm{KM}}=\left(R^{*}\right)^{n / n+1}$.

Proof. Using Lemma 1,

$$
\begin{aligned}
R_{\mathrm{KM}} & =\frac{(-1)^{n}|U|}{\left\{(-1)^{n} \lambda^{2}|S|\right\}^{n /(n+1)}}=\frac{(-1)^{n}|U|}{\left[(-1)^{n}\left\{-|U| \cdot \tilde{u}^{\prime} U^{-1} \tilde{u}\right\}\right]^{n /(n+1)}} \\
& =\left\{\frac{(-1)^{n}|U|}{\left(-\tilde{u}^{\prime} U^{-1} \tilde{u}\right)^{n}}\right\}^{1 /(n+1)}=\left(R^{*}\right)^{n /(n+1)}
\end{aligned}
$$

All our measures of absolute risk aversion are obtained by normalising $\tilde{u}^{\prime} U^{-1} \tilde{u}$ in such a way that the resulting measure is free of the monetary as well as the utility units. Theorem 3 provides a direct and general proof that the Khilstrom-Mirman measure is also related to such a process of normalising $\tilde{u}^{\prime} U^{-1} \tilde{u}$ and the logic of the choice of Hessian determinant as the normaliser is explained through the construction of $R^{*}$. The observation that $\tilde{u}^{\prime} U^{-1} \tilde{u}$ plays such a crucial role in all scalar measures of risk aversion seems to be quite intriguing. Finally, it should be noted that in the multidimensional case, the measures were designed to compare global risk averseness of two individuals. We may use any of these measures to examine the concavity of $g$ and, as necessary and sufficient conditions, they are equivalent. However, the relative suitability of a particular measure will depend upon the form of the utility functions under consideration and is a matter of computational convenience.

\section{REFERENCES}

1. K. J. ARrow, "Essays in the Theory of Risk Bearning," Markham, Chicago, 1971.

2. R. Deschamps, Risk aversion and demand functions, Econometrica 41 (1973) 455-466.

3. G. T. Duncan, A matrix measure of multivariate local risk aversion, Econometrica 45 (1977), 895-903.

4. G. HANOCH, Risk aversion and consumer preferences, Econometrica 45 (1977), 413-426.

5. E. KARNI, On multivariate risk aversion, Econometrica 47 (1979), 1391-1401.

6. R. E. KHILstrom and L. J. Mirman, Risk aversion with many commodities, J. Econ. Theory 8 (1974), 361-388. 


\section{BISWAS Measures of Risk Aversion}

7. J. W. Pratt, Risk aversion in the small and in the large, Econometrica 32 (1964), 122-136.

8. M. Rothschild AND J. E. Stiglitz, Increasing risk: I, A definition, J. Econ. Theory 2 (1970), 225-243.

9. J. E. Stiglitz, Behaviour towards risk with many commodities, Econometrica 37 (1969), $660-667$.

Originally published in Journal of Economic Theory (1983), 29, 347-352 